\title{
Pensare storicamente. Didattica, laboratori, manuali
}

di Nadia Olivieri

Giu 9, 2021 | In evidenza, Uno sguardo su $\mid \underline{0 \mid}$

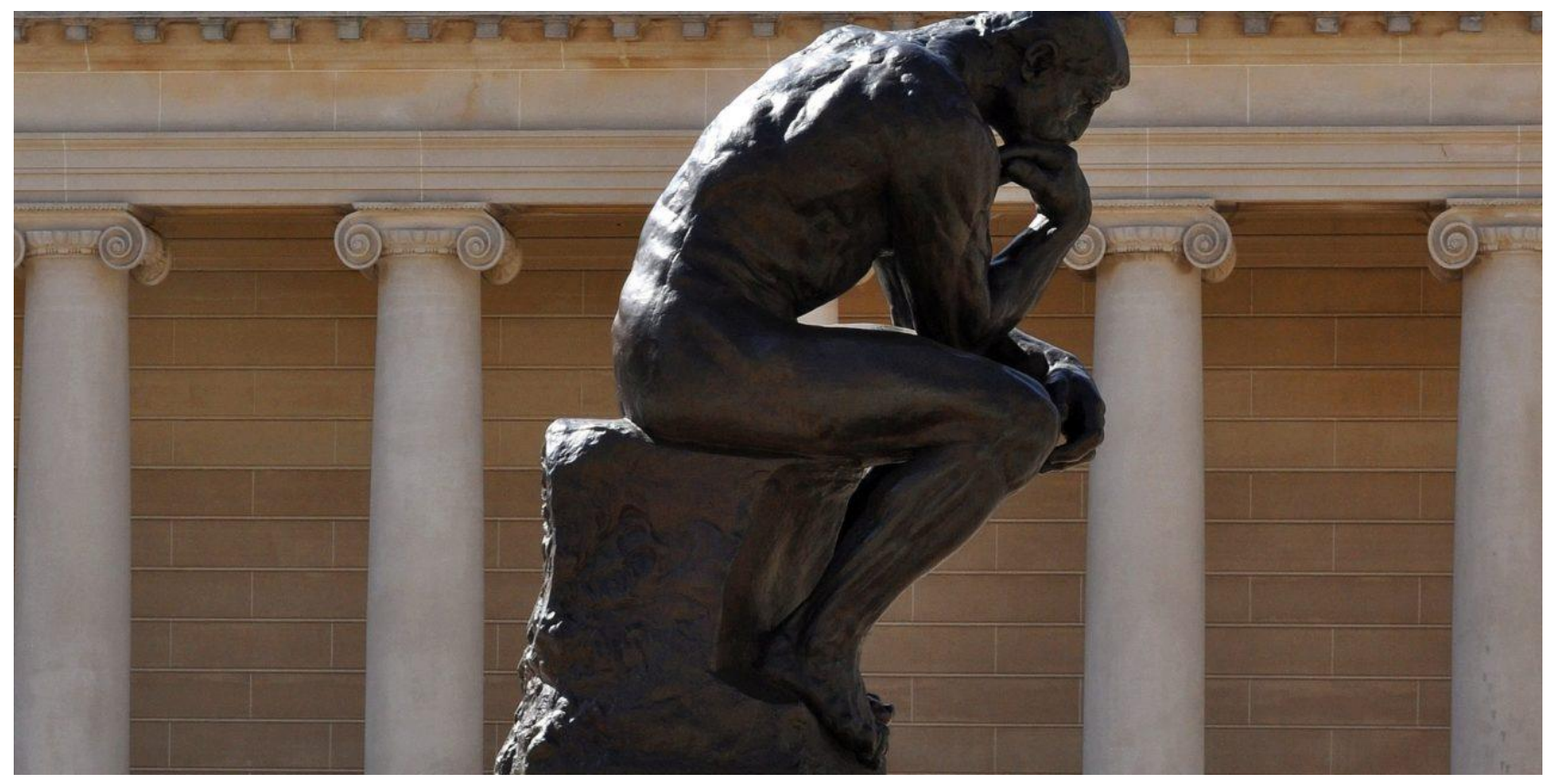

“Il pensatore" di Auguste Rodin. Scultura conservata al Museo Rodin di Parigi

\section{Salvatore Adorno, Luigi Ambrosi, Margherita Angelini (a cura di) Pensare storicamente. Didattica, laboratori, manuali Franco Angeli, Milano 2020}

È già da qualche anno che le associazioni dei docenti e ricercatori di storia si stanno occupando di questioni didattiche e, nell'ambito del Coordinamento delle società storiche, hanno formato una apposita Commissione che si interroga sul rapporto tra ricerca e didattica e tra università e scuola. Dal 2017 al 2019 si sono svolti ben tre convegni nazionali: il primo, a Roma, su Insegnare la storia ai "Millennials", 27 gennaio 2017; il secondo, a Verona, su Insegnare storia oggi nella scuola secondaria, 22-23 febbraio 2018 e il terzo a Siracusa, dal titolo Lezioni di storia. Tecniche ed esperienza di didattica frontale, 12-13 aprile 2019. Alcuni contributi di quegli appuntamenti sono stati ora pubblicati in questo volume, che raccoglie le voci di storici accademici e di docenti di diversi ordini di scuola, riuniti a ragionare insieme non solo su pratiche didattiche e saperi disciplinari, ma sul senso stesso dell'insegnamento della storia e sul suo possibile contributo alla formazione degli studenti.

Il libro è organizzato in sei diverse sezioni: la didattica, i metodi, le risorse, i manuali, la formazione e le discipline. Vi si trovano contributi di grande interesse, che toccano tutti gli aspetti della didattica della storia, a partire dal "cosa" e "come" insegnarla a scuola. Chiara l'indicazione metodologica: solo una didattica di tipo laboratoriale porta a sintesi virtuosa la sterile disputa fra "conoscenze" e "competenze". Se le competenze storiche sono abilità "agite" - ci dice Salvatore 
Adorno - sono i laboratori che possono svilupparle. Eppure l'approccio laboratoriale - di cui Antonio Brusa ripercorre la storia dagli anni Settanta in avanti - non è ancora riuscito ad affermarsi nelle nostre scuole. Ad ostacolarlo concorrono la necessità di dover operare a monte una drastica revisione del curricolo verticale, una selezione dei contenuti più adatti all'approccio e una programmazione "a ritroso", che liberi gli ultimi mesi dei due cicli scolastici per affrontare la contemporaneità vera, quella del "tempo presente". Tentativi virtuosi sono in corso (per esempio con la rete di LabSto21 ricordata da Luigi Ambrosi), ma si scontrano con l'apparentemente inscalfibile inerzia nel riproporre un insegnamento tradizionale, basato su un canone narrativo consolidato e costante (sicuramente rassicurante per i docenti), cristallizzato nei manuali scolastici. Sconsolanti le analisi che ne emergono dai saggi di Miccichè, Rovinello, Brusa, Loré. Narrazione storiografica vecchia, stereotipi e periodizzazioni superate, apparati documentari relegati ad “eserciziari”, iconografia posta solo a mo' di illustrazione: i manuali rimandano immediatamente ad un'idea di didattica della storia basata sulla classica lezione frontale. Nulla che miri ad appassionare gli studenti.

Di ben altro respiro gli esempi didattici e le riflessioni riportati nei saggi di Angelini, Ambrosi, Ciappetta, La Manna, che fanno emergere la praticabilità di un insegnamento della storia globale e inter-disciplinare, capace di ricorrere a metodologie e strumenti che siano in grado di coinvolgere $\mathrm{i}$ ragazzi: archivi simulati, EAS (esperienze di apprendimento situato), studi di caso, scrittura di voci di Wikipedia, debate, giochi, uscite didattiche, percorsi iconografici e filmografici, attività svolte in gruppi cooperativi e con sperimentazioni di CLIL. Sono davvero numerosi gli spunti offerti, con anche molti rimandi ad articoli di Novecento.org, indicati come esempi virtuosi. Didattica "innovativa" anche a prescindere dall'uso delle tecnologie digitali, che spesso mascherano la classica lezione frontale impartita con nuovi mezzi.

Ma se la possibilità di innovare è così ricca resta da chiedersi quale sia il motivo del persistere di tante resistenze a modificare pratiche consolidate. È probabile che le ragioni affondino le loro radici nella preparazione universitaria dei docenti, spesso provenienti da percorsi di studio relativi ad altre materie (filosofia e italiano in primis), con modalità di accesso all'insegnamento troppe volte modificate negli ultimi anni e con una formazione in servizio di carattere ancora volontario. Una situazione ben fotografata da Bianchi nel suo saggio. Il singolo docente spesso si sente solo: «manca un pensiero didattico condiviso e strutturato»[1], lamenta Marcellini nel suo contributo su didattica della storia e risorse digitali, e, nel mare magnum del web, mancano strumenti rivolti alla didattica. Le banche-dati online, infatti, se ben si prestano alle necessità della ricerca, sono di utilizzo problematico per utenti inesperti quali sono i nostri studenti.

Inesperti sì, perché dietro l'apparente dimestichezza con i device tecnologici, si cela l'incapacità di gestire la mole enorme di risorse che la rete nasconde. Serve un'educazione alla cittadinanza digitale per comprendere autenticità, provenienza, manipolazione delle informazioni (le fonti).

E proprio «tempo e fonti [aggiungerei, spazio] sono i pilastri su cui costruire una didattica della storia attiva e non meramente trasmissiva»[2], ci ricorda Salvatore Adorno. Una storia che alleni i ragazzi a pensare storicamente, secondo il felice sintagma scelto come titolo del libro, tramite laboratori che permettano di «analizzare frammenti di realtà, passata o presente, attraverso l'analisi, l'inferenza, la discussione, la ricerca, la concettualizzazione»[3] e che insegnino a «orientarsi, selezionare, distinguere e valutare le informazioni» (secondo le indicazioni di Girardet richiamate da Ambrosi[4]).

Non sfugge il ruolo fondamentale che alla storia viene attribuito nel "nuovo" insegnamento di educazione civica. 
Nella nuova globital [sintesi di global e digital, n.d.r.] public sphere circolano narrazioni del passato caratterizzate da processi di decontestualizzazione (per produrre usi svariati), despazializzazione (il nuovo spazio "virtuale" offerto dai media), moralizzazione (per emozionare e mobilitare), fondamentalizzazione[5]

spiega Villani nel suo imperdibile saggio sul rapporto fra didattica della storia e public history, di cui è necessario rendere i ragazzi consapevoli. Un compito che la scuola si deve assumere e che ha un'evidente ricaduta sulla formazione dei nostri studenti, i veri cittadini del futuro.

Solo rendendo «gli studenti protagonisti attivi dell'uso e della costruzione di narrative sensate e consapevoli», possiamo restituire loro non solo la possibilità di legare presente e passato, ma di immaginare il futuro. Nella consapevolezza che, in fondo, - ci ricorda ancora Adorno - sono queste narrative a dare «senso all'esistenza umana, aiutando la formazione dell'identità individuale e collettiva»[6].

In sintesi è forse questa la lezione più "alta" che un insegnante può trarre dalla lettura di questo ricchissimo libro che non è di didattica della storia, ma apre a una riflessione sulla didattica della storia e, in qualche modo, anche alla dimensione epistemologica della disciplina. Peccato che, nell'anno dell'emergenza pandemica, Pensare storicamente rischi di passare sotto silenzio, perché è un testo che non dovrebbe mancare nella biblioteca degli insegnanti della disciplina.

Note:

[1] C. Marcellini, Didattica della storia e risorse digitali. Dalla pratica quotidiana ai problemi aperti, in Adorno S., Ambrosi L., Angelini M. (a cura di), Pensare storicamente. Didattica, laboratori, manuali, Franco Angeli, Milano 2020, p. 171.

[2] S. Adorno, Pensare la didattica della storia, ivi, p. 19.

[3] A. Miccichè, Il sussidiario di storia e la Scuola primaria, ivi, p. 217.

[4] L. Ambrosi, Bisogni educativi degli alunni nell'apprendimento della storia, ivi, p. 87.

[5] C. Villani, Public history e didattica della storia nell'età delle history wars, ivi, p. 200.

[6] S. Adorno, Pensare la didattica della storia, ivi, p. 20. 